\title{
Hepatocellular Carcinoma Is the Most Frequent Final Diagnosis of Focal Liver Lesions Identified in a Cross-Sectional Evaluation of Patients with Chronic Liver Disease in Saudi Arabia
}

\author{
Adnan Agha, ${ }^{1,2}$ Manuele Furnari, ${ }^{3}$ Rafaat Morched Chakik, ${ }^{1}$ Mamdouh M. Abdulhadi Ali, ${ }^{1}$ \\ Dib Alsaudi, ${ }^{1}$ Mohammed Bazeed, ${ }^{4}$ Vincenzo Savarino, ${ }^{3}$ and Edoardo G. Giannini ${ }^{3}$ \\ ${ }^{1}$ Department of Medicine, Armed Forces Hospital Southern Region, Khamis Mushayt, Saudi Arabia \\ ${ }^{2}$ Department of Medicine, Jersey General Hospital, The Parade, St. Helier, Jersey, Channel Isles JE1 3QS, UK \\ ${ }^{3}$ Gastroenterology Unit, Department of Internal Medicine, IRCCS Azienda Ospedaliera Universitaria San Martino-IST, \\ University of Genoa, 16132 Genoa, Italy \\ ${ }^{4}$ Department of Radiology, King Fahad Hospital, Armed Forces Hospital Southern Region, Khamis Mushayt, Saudi Arabia \\ Correspondence should be addressed to Edoardo G. Giannini; egiannini@unige.it
}

Received 17 September 2014; Accepted 16 December 2014

Academic Editor: Daizo Yoshida

Copyright (c) 2015 Adnan Agha et al. This is an open access article distributed under the Creative Commons Attribution License, which permits unrestricted use, distribution, and reproduction in any medium, provided the original work is properly cited.

\begin{abstract}
Background. Hepatocellular carcinoma (HCC) is a frequent diagnosis in patients with chronic liver disease (CLD) and a newly identified liver lesion, although benign diseases may also be responsible for this finding. Objective. To evaluate the characteristics of focal liver lesions in a population of patients with CLD not under surveillance for HCC in the Middle East. Methods. We performed a cross-sectional study evaluating 77 patients with CLD and a focal liver lesion identified during ultrasonography. Patients' characteristics were analyzed on the basis of the final diagnosis (HCC versus benign lesions). Results. The most frequent diagnosis was HCC (64.9\%). These patients were older (median age 64 versus 55 years, $P=0.003$ ) and cirrhotics $(80.0 \%$ versus $51.9 \%, P=0.018)$, with multinodular lesions $(58.0 \%$ versus $29.6 \%, P=0.031)$ and portal vein thrombosis $(24.0 \%$ versus $0 \%$, $P=0.001)$ compared to patients with benign lesions. Prevalence of elevated alpha-fetoprotein $(>10 \mathrm{ng} / \mathrm{mL})$ was similar in both groups ( $80.0 \%$ versus $88.9 \%, P=0.198$ ). Cirrhosis (odds ratio: 3.283 ) and multinodularity (odds ratio: 2.898 ) were independently associated with HCC. Conclusions. HCC is the most common diagnosis in Middle-Eastern patients with CLD and a liver lesion identified outside HCC surveillance programs, especially in cirrhotic patients. In these patients, elevated alpha-fetoprotein does not differentiate HCC from benign lesions.
\end{abstract}

\section{Introduction}

Hepatocellular carcinoma (HCC) is responsible for approximately $6 \%$ of all human cancers $[1,2]$. Typically, HCC develops in patients with chronic liver disease and cirrhosis in whom chronic hepatitis B virus (HBV) and hepatitis C virus (HCV) infection and alcohol consumption are the most common etiological factors for liver disease [3, 4]. Surveillance programs based on liver ultrasound are the cornerstone of early detection of HCC, and they are able to improve patients prognosis due to better staging at diagnosis and a greater likelihood of receiving curative treatments $[5,6]$. Although the majority of focal liver lesions detected in a cirrhotic liver during cross-sectional imaging studies ultimately prove to be HCC, some other lesions-either benign or malignant-can be observed in these patients. Indeed, focal liver lesions may be heterogeneous andbesides HCC-dysplastic nodules, hemangioma, cysts, and lymphoma may be the final diagnosis, and the incidence of these various lesions may vary according to population and geographic area [7-13].

In this study, carried out in a population of patients residing in a Middle Eastern country, our aim was to conduct a cross-sectional evaluation analyzing the characteristics and final diagnosis of hepatic focal lesions identified in a series of unselected patients with chronic liver disease who underwent liver ultrasound evaluation. 


\section{Materials and Methods}

We performed a cross-sectional study of patients with chronic liver disease undergoing liver ultrasound at the Department of Medicine, King Fahad Hospital, Armed Forces Hospital Southern Region, Khamis Mushait, Saudi Arabia, and where focal hepatic lesions were identified. Patients who were on regular surveillance for HCC were not included in this study. Recent (within 6 months of inclusion) radiological evaluation or hepatic/abdominal surgery, presence of known primary tumor elsewhere, and history of recent sepsis were considered exclusion criteria.

Focal hepatic lesions identified at ultrasonography were further evaluated by means of contrast-enhanced computed tomography, magnetic resonance imaging, or lesion biopsy until a final diagnosis was reached. Liver function tests, HBV and HCV serology, serum alpha-fetoprotein levels, and antiSchistosoma antibodies titer were recorded in all patients. Diagnosis of cirrhosis was based on patients history, clinical and radiological evaluation, or histology.

2.1. Statistical Analysis. Continuous variables are expressed as median and range and categorical variables as absolute number and proportion. One-way analysis of variance for nonparametric data was performed by post hoc analysis using Kruskal-Wallis $H$ test, parametric variables were compared by post hoc analysis using Tukey test, and Fisher's exact test was used to compare categorical variables. Multivariate logistic regression analysis (forward method) was carried out on parameters significantly associated with HCC diagnosis in univariate analysis. Data analyses were performed on MedCalc software (MedCalc software, Acacialaan 22, 8400 Ostend, Belgium).

\section{Results}

Demographic and biochemical characteristics of the 77 patients who made up the study population are shown in Table 1. Etiology ofliver disease was infection with hepatitis viruses in 30 patients (39.0\%): HBV in 22 (28.6\%) and HCV in 8 (10.4\%). Features consistent with a diagnosis of cirrhosis were observed in 54 patients (70.1\%) and among them $24(44.4 \%)$ had ascites, which was clinically evident in 2 patients $(8.3 \%)$ and instrumentally detected alone in 22 patients $(91.7 \%)$.

Forty patients (51.9\%) had 1 nodule, 9 patients (11.7\%) had 2-4 nodules, and 26 patients $(33.8 \%)$ had $\geq 5$ nodules, and 2 patients (2.6\%) had infiltrative lesions (Figure 1). Diagnostic work-up led to the diagnosis of HCC in 50 patients (64.9\%), hepatic hemangioma in 13 patients (16.9\%), regenerative nodules in 8 patients $(10.4 \%)$, complex hydatid cyst in 2 patients $(2.6 \%)$, focal fat sparing in 2 patients $(2.6 \%)$, and simple cyst in 2 patients 1 (2.6\%). Presence of complete or partial portal vein thrombosis was found in 12 patients (15.6\%): 5 affecting the main portal vein trunk, 5 affecting the right intrahepatic branch, 1 affecting the left intrahepatic branch, and 1 extending to the middle and right hepatic veins and the superior vena cava.
TABLE 1: Main demographic and biochemical characteristics of the 77 study patients.

\begin{tabular}{lcc}
\hline Variable & Unit & Value \\
\hline Gender & Male & $53(68)$ \\
Age & Years & $64(29-90)$ \\
Alanine aminotransferase & $\mathrm{IU} / \mathrm{mL}$ & $48(14-892)$ \\
Aspartate aminotransferase & $\mathrm{IU} / \mathrm{mL}$ & $62(10-953)$ \\
Gamma-glutamyl transpeptidase & $\mathrm{IU} / \mathrm{mL}$ & $110(12-2,020)$ \\
Alkaline phosphatase & $\mathrm{IU} / \mathrm{mL}$ & $133(44-2,331)$ \\
Alpha-fetoprotein & $\mathrm{ng} / \mathrm{mL}$ & $14.5(1.2-144,985)$ \\
Total bilirubin & $\mu \mathrm{mol} / \mathrm{L}$ & $28(3-459)$ \\
Albumin & $\mathrm{gr} / \mathrm{dL}$ & $2.7(1.4-2.7)$ \\
INR & $\mathrm{Ratio}$ & $1.2(1-7.5)$ \\
\hline
\end{tabular}

Data are shown as absolute value and percentage or median and range.

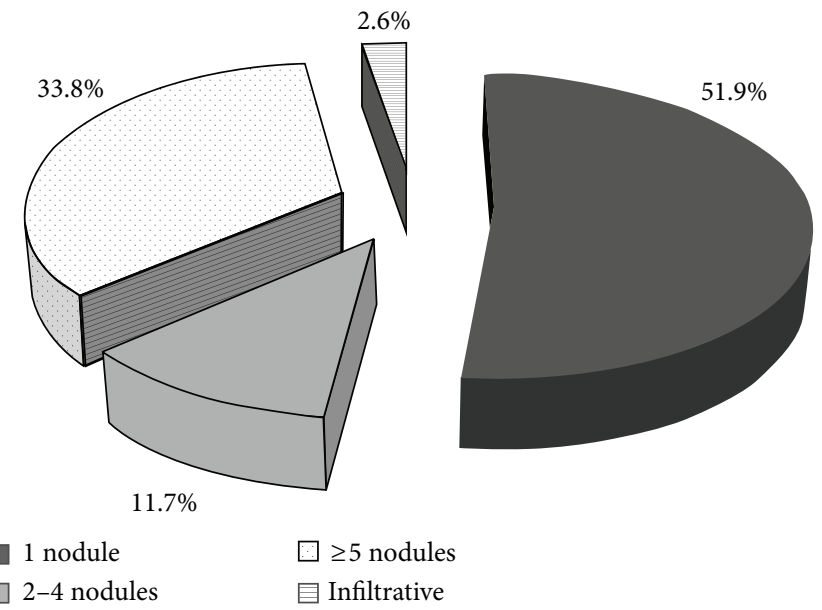

Figure 1: Proportion of patients with single, multinodular, or invasive focal liver lesions identified at imaging.

According to the final diagnosis of the hepatic lesions (HCC versus benign lesions), we further subdivided our population into 2 groups and evaluated their main demographic and clinical characteristics (Table 2). Patients with HCC were older and presence of cirrhosis, ascites, and portal vein thrombosis was observed more frequently in these patients as compared to patients with benign lesions. Although serum alpha-fetoprotein levels were significantly higher in patients with HCC $(P=0.001)$, the proportion of patients with alpha-fetoprotein levels $>10 \mathrm{ng} / \mathrm{mL}$ was not significantly different in the two groups $(P=0.525)$. Positivity for Schistosoma antibodies was numerically more frequent in patients with HCC (20\% versus $7.4 \%, P=0.198)$. Lastly, in multivariate logistic regression analysis, presence of cirrhosis (odds ratio: 3.283 (95\% confidence interval, 1.141-9.450)) and multinodularity (odds ratio 2.898 (95\% confidence interval, 1.032-8.142)) were the parameters independently associated with HCC diagnosis. 
TABLE 2: Main demographic and characteristics of the study population subdivided according to the final diagnosis of the hepatic lesions.

\begin{tabular}{|c|c|c|c|c|}
\hline & & $\mathrm{HCC}(n=50)$ & Benign $(n=27)$ & $P$ value \\
\hline Gender & Male & $37(74.0 \%)$ & $16(59.3 \%)$ & 0.206 \\
\hline Age & Years & $64(29-86)$ & $55(29-50)$ & 0.003 \\
\hline Viral etiology & Yes & $22(44.0 \%)$ & $8(29.6 \%)$ & 0.234 \\
\hline Cirrhosis & Yes & $40(80.0 \%)$ & $14(51.9 \%)$ & 0.018 \\
\hline Ascites & Yes & $20(40.0 \%)$ & $4(14.8 \%)$ & 0.038 \\
\hline Number of lesions & $>1$ & $29(58.0 \%)$ & $8(29.6 \%)$ & 0.031 \\
\hline Portal vein thrombosis & Yes & $12(24.0 \%)$ & $0(0 \%)$ & 0.001 \\
\hline Alpha-fetoprotein & $\mathrm{ng} / \mathrm{mL}$ & $61.1(1.2-144,985)$ & $11.9(8.7-16.5)$ & $<0.001$ \\
\hline Alpha-fetoprotein $>10 \mathrm{ng} / \mathrm{mL}$ & Yes & $40(80.0 \%)$ & $24(88.9 \%)$ & 0.525 \\
\hline Schistosoma antibodies & Positive & $10(20.0 \%)$ & $2(7.4 \%)$ & 0.198 \\
\hline
\end{tabular}

Data are shown as absolute value and percentage or median and range. HCC: hepatocellular carcinoma.

\section{Discussion}

Patients affected by chronic liver disease carry a higher risk of developing HCC as compared to the general population, and in patients with cirrhosis this risk is high enough to mandate screening and surveillance for the detection of early tumors [2, 6]. However, other malignant or benign diseases may be responsible for the presence of focal liver lesions in patients with chronic liver disease [7]. In this crosssectional study, carried out in a population of patients with chronic liver disease residing in the Middle East, we found that approximately two-thirds of newly identified focal liver lesions were due to HCC and that the proportion of HCC was even higher (i.e., 80\%) in patients with liver cirrhosis. As expected in our geographical area, the patients population was mainly made up of subjects with viral etiology of liver disease, and the majority of patients had advanced liver disease $[14,15]$.

We observed that patients with HCC were older and had more frequently liver cirrhosis and clinical features of portal hypertension thus confirming that HCC tends to develop in patients with more advanced liver disease. Patients with HCC also had more frequently a multinodular disease and portal vein thrombosis: although this study evaluated only patients who underwent a cross-sectional imaging study and did not include patients on regular follow-up, we feel that these advanced features may be attributable to the low uptake of HCC surveillance in our geographical area [16]. Furthermore, although patients with HCC had significantly higher median alpha-fetoprotein levels, the proportion of patients with serum alpha-fetoprotein $>10 \mathrm{ng} / \mathrm{mL}$ was not different as compared to patients with benign lesions. This finding underscores the poor performance of this marker also in Middle Eastern patients with prevalently chronic viral liver disease and further emphasizes the poor sensitivity of altered alpha-fetoprotein levels when its performance is assessed on the "background noise" of cirrhosis, advanced liver disease, and altered aminotransferases [17-21]. In keeping with clinical and experimental studies suggesting that infection with Schistosoma may be a further risk factor for the presence of
HCC, we observed a numerically greater prevalence of Schistosoma antibodies positivity in patients with HCC although the difference with patients harboring benign lesions was not statistically significant likely due to the relatively small cohort evaluated [22, 23]. Lastly, only presence of cirrhosis and multinodularity were the parameters that were independently associated with a final diagnosis of HCC.

Due to the current and estimated burden of chronic liver disease in the Middle East, the limited access to potential treatment, and the increasing epidemic of some diseases such as nonalcoholic fatty liver disease coupled with aging of the population, it is expected that in the future the proportion of patients with advanced liver disease diagnosed with HCC in our geographical area will likely increase [24, 25]. In this regard, it is interesting to note that, besides the presence of cirrhosis and multinodularity, there were no other features able to pinpoint patients with chronic liver disease and focal liver lesions who were more likely to have a malignant disease. On these bases, it is important that programs aimed at the early detection of HCC in cirrhotic patients be implemented in our region so as to detect lesions that can be effectively cured [2, 6, 26, 27].

This study has some limitations such as the lack of information on the possible pretest suspicion of HCC in some patients (e.g., weight loss and worsening of ascites) and on the patients outcome as patients HCC management was carried out at a different facility.

In conclusion, in this cross-sectional study we observed that the majority of focal liver lesions identified in a population with prevalent liver cirrhosis in the Middle East can be attributable to HCC. This result calls for implementation of screening and surveillance programs for early HCC detection in our geographical area.

\section{Conflict of Interests}

The authors declare that there is no conflict of interests regarding the publication of this paper. 


\section{References}

[1] S. Mittal and H. B. El-Serag, "Epidemiology of hepatocellular carcinoma: consider the population," Journal of Clinical Gastroenterology, vol. 47, no. 1, pp. S2-S6, 2013.

[2] L. Bolondi, U. Cillo, M. Colombo et al., "Position paper of the Italian Association for the Study of the Liver (AISF): the multidisciplinary clinical approach to hepatocellular carcinoma," Digestive and Liver Disease, vol. 45, no. 9, pp. 712-723, 2013.

[3] V. Santi, D. Buccione, A. di Micoli et al., "The changing scenario of hepatocellular carcinoma over the last two decades in Italy," Journal of Hepatology, vol. 56, no. 2, pp. 397-405, 2012.

[4] F. Trevisani, M. C. Cantarini, J. R. Wands, and M. Bernardi, "Recent advances in the natural history of hepatocellular carcinoma," Carcinogenesis, vol. 29, no. 7, pp. 1299-1305, 2008.

[5] V. Santi, F. Trevisani, A. Gramenzi et al., "Semiannual surveillance is superior to annual surveillance for the detection of early hepatocellular carcinoma and patient survival," Journal of Hepatology, vol. 53, no. 2, pp. 291-297, 2010.

[6] E. G. Giannini, A. Cucchetti, V. Erroi, F. Garuti, F. Odaldi, and F. Trevisani, "Surveillance for early diagnosis of hepatocellular carcinoma: how best to do it?" World Journal of Gastroenterology, vol. 19, no. 47, pp. 8808-8821, 2013.

[7] E. Caturelli, F. Bartolucci, E. Biasini et al., "Diagnosis of liver nodules observed in chronic liver disease patients during ultrasound screening for early detection of hepatocellular carcinoma," The American Journal of Gastroenterology, vol. 97, no. 2, pp. 397-405, 2002.

[8] J. E. Naschitz, E. Zuckerman, N. Elias, and D. Yeshurun, "Primary hepatosplenic lymphoma of the B-cell variety in a patient with hepatitis C liver cirrhosis," The American Journal of Gastroenterology, vol. 89, no. 10, pp. 1915-1916, 1994.

[9] E. Caturelli, S. Fusilli, L. Costarelli et al., "Focal ultrasound lesions in cirrhotic liver diagnosed as regenerative nodules by biopsy: a morphometric analysis," Journal of Clinical Gastroenterology, vol. 17, no. 1, pp. 67-72, 1993.

[10] F. Fornari, C. Filice, G. L. Rapaccini et al., "Small $(\leq 3 \mathrm{~cm})$ hepatic lesions: results of sonographically guided fine-needle biopsy in 385 patients," Digestive Diseases and Sciences, vol. 39, no. 10, pp. 2267-2275, 1994.

[11] K. Seitz, C. Greis, A. Schuler et al., "Frequency of tumor entities among liver tumors of unclear etiology initially detected by sonography in the noncirrhotic or cirrhotic livers of 1349 patients: results of the DEGUM multicenter study," Ultraschall in der Medizin, vol. 32, no. 6, pp. 598-603, 2011.

[12] J. Blessmann, N. D. Khoa, L. V. An, and E. Tannich, "Ultrasound patterns and frequency of focal liver lesions after successful treatment of amoebic liver abscess," Tropical Medicine and International Health, vol. 11, no. 4, pp. 504-508, 2006.

[13] K. R. Reddy, "Benign and Solid tumors of the liver: relationship to sex, age, size of tumors, and outcome," American Surgeon, vol. 67, no. 2, pp. 173-178, 2001.

[14] E. A. Ayoola and M. O. Gadour, "Hepatocellular carcinoma in Saudi Arabia: Role of hepatitis B and C infection," Journal of Gastroenterology and Hepatology, vol. 19, no. 6, pp. 665-669, 2004.

[15] B. Fashir, V. Sivasubramaniam, S. Al Momen, and H. Assaf, "Pattern of liver disease in a Saudi patient population: a decade of experience at security forces hospital, Riyadh, KSA," Saudi Journal of Gastroenterology, vol. 2, pp. 50-52, 1996.

[16] A. Fani, I. Fani, B. Eshratie, P. Samadian, P. Fani, and Y. Gorishi, "Screening for hepatocellular carcinoma in hepatitis B and C chronic carriers in Iran," Indian Journal of Gastroenterology, vol. 26, no. 6, pp. 297-298, 2007.

[17] F. M. Sanai, S. Sobki, K. I. Bzeizi et al., "Assessment of alphafetoprotein in the diagnosis of hepatocellular carcinoma in Middle Eastern patients," Digestive Diseases and Sciences, vol. 55, no. 12, pp. 3568-3575, 2010.

[18] E. G. Giannini, F. Farinati, and F. Trevisani, "Alpha-fetoprotein in hepatocellular carcinoma surveillance: wake not the dead," Hepatology, vol. 54, no. 1, pp. 376-377, 2011.

[19] E. G. Giannini, V. Erroi, and F. Trevisani, "Effectiveness of $\alpha$-fetoprotein for hepatocellular carcinoma surveillance: the return of the living-dead?" Expert Review of Gastroenterology and Hepatology, vol. 6, no. 4, pp. 441-444, 2012.

[20] M. Sherman, "Current status of alpha-fetoprotein testing," Gastroenterology and Hepatology, vol. 7, no. 2, pp. 113-114, 2011.

[21] E. G. Giannini, G. Sammito, F. Farinati et al., "Determinants of alpha-fetoprotein levels in patients with hepatocellular carcinoma: implications for its clinical use," Cancer, vol. 120, pp. 2150-2157, 2014.

[22] M. M. El-Tonsy, H. M. Hussein, T. E.-S. Helal, R. A. Tawfik, and K. M. Koriem, "Schistosoma mansoni infection: is it a risk factor for development of hepatocellular carcinoma?" Acta Tropica, vol. 128, no. 3, pp. 542-547, 2013.

[23] M. A. Hamed and S. A. Ali, "Non-viral factors contributing to hepatocellular carcinoma," World Journal of Hepatology, vol. 5, no. 6, pp. 311-322, 2013.

[24] A. A. Abdo, F. M. Sanai, and F. Z. Al-Faleh, "Epidemiology of viral hepatitis in Saudi Arabia: are we off the hook," Saudi Journal of Gastroenterology, vol. 18, no. 6, pp. 349-357, 2012.

[25] M. H. Ismail, "Nonalcoholic fatty liver disease and type 2 diabetes mellitus: the hidden epidemic," The American Journal of the Medical Sciences, vol. 341, no. 6, pp. 485-492, 2011.

[26] E. G. Giannini and F. Trevisani, "Surveillance for hepatocellular carcinoma: just do it!, The American Journal of Gastroenterology, vol. 108, no. 6, pp. 1013-1014, 2013.

[27] E. G. Giannini, F. Farinati, and F. Trevisani, "Adequate interval for hepatocellular carcinoma surveillance," American Journal of Gastroenterology, vol. 108, no. 1174, 2013. 


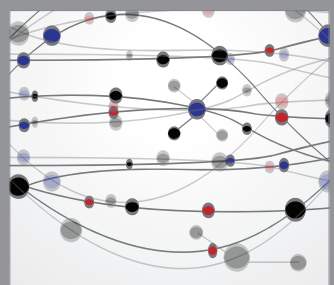

The Scientific World Journal
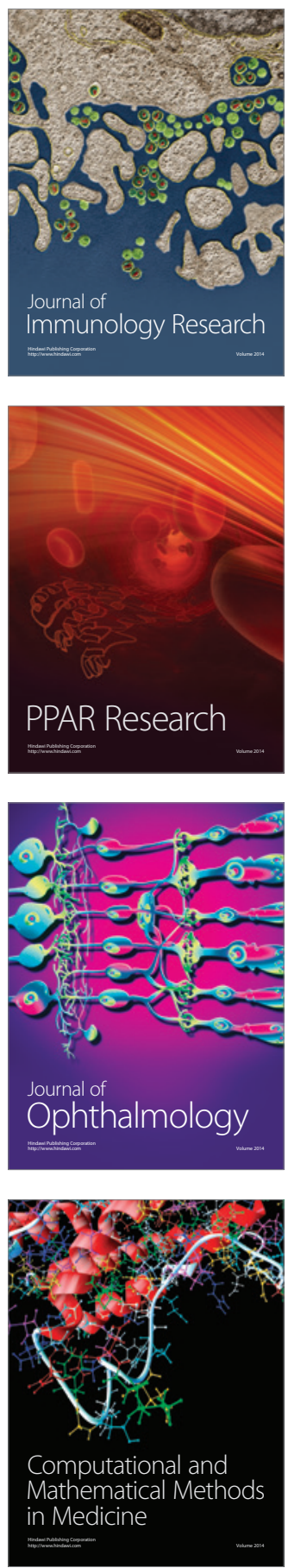

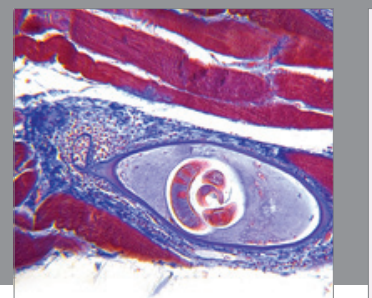

Gastroenterology

Research and Practice
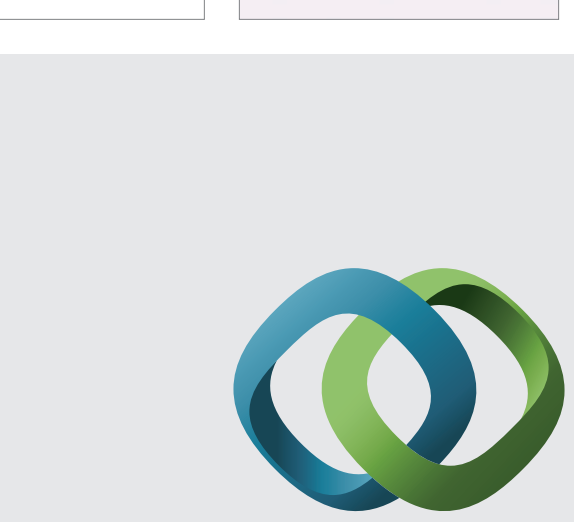

\section{Hindawi}

Submit your manuscripts at

http://www.hindawi.com
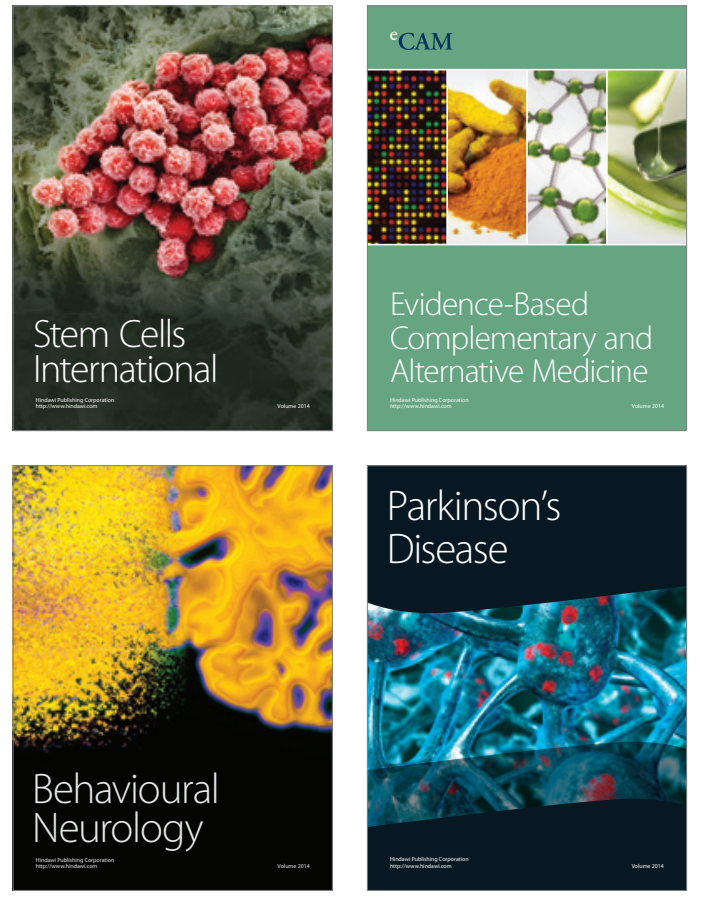
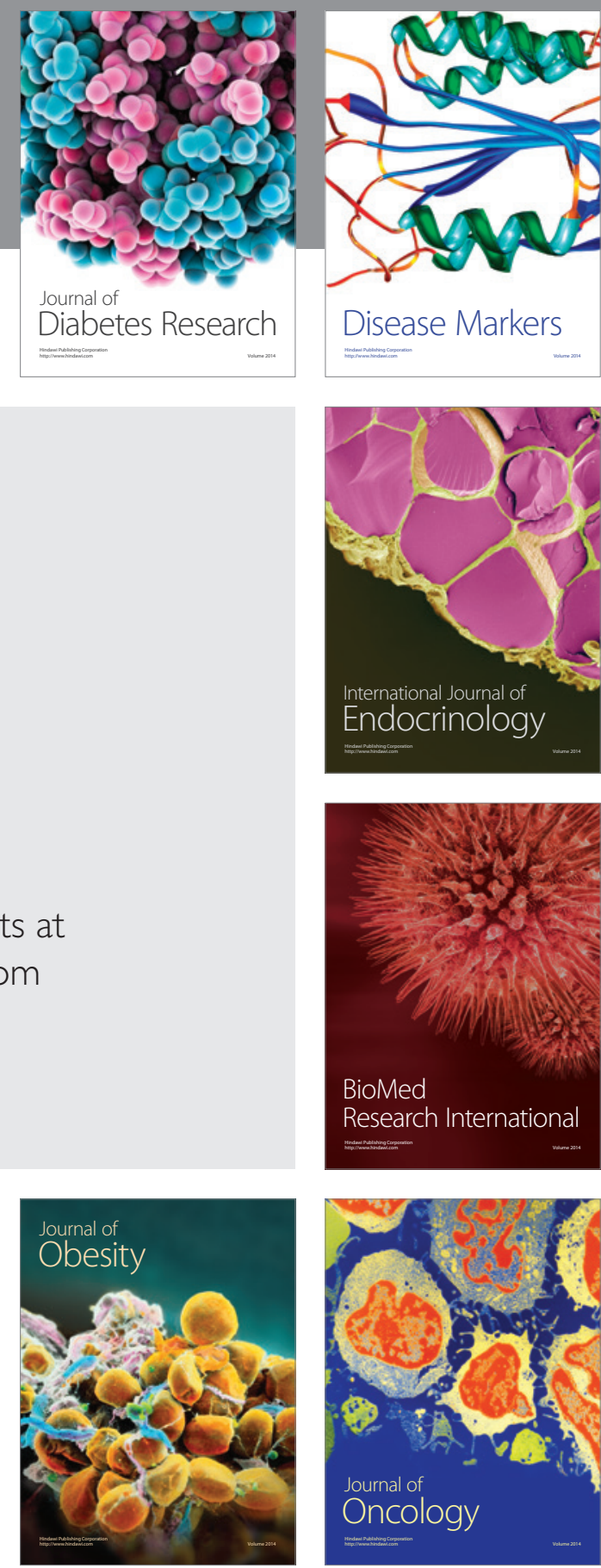

Disease Markers
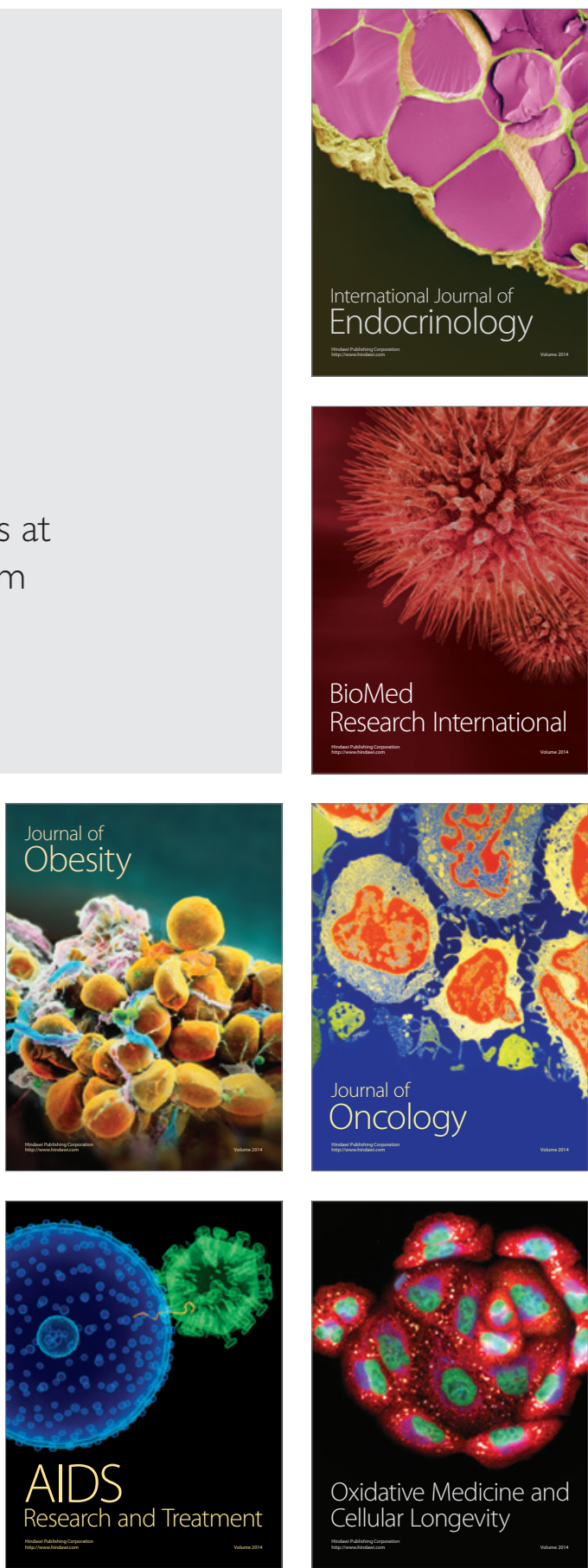